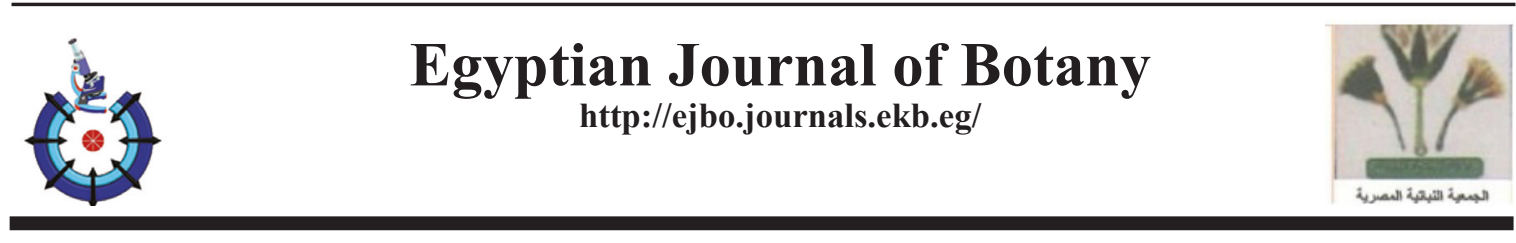

\title{
Egyptian Fossil Angiosperm Wood: A Guide to their Anatomical Identification
}

Zainab M. El-Noamani

El-Saadawi Lab., Botany Department, Faculty of Science, Ain Shams University, Cairo, 11566, Egypt.

\begin{abstract}
FEW of the existing publications on Egyptian fossil plants provide special aids to identifying fossil wood remains. Two dichotomous keys to the angiosperm petrified wood species reported to date from the Egyptian strata are presented here to facilitate the tentative identification of angiosperm fossil wood specimens. The first key includes 19 species of Palmoxylon which is the morphogenus for the petrified palm stem that is the common representative of monocotyledones (Arecaceae) in the Egyptian fossil flora and can be identified to the species level. The second key contains 46 species of dicotyledonous petrified wood of different families reported in the literature from different ages and locations in Egypt. These keys would benefit palaeobotanists who might find the literature on Egyptian fossil wood not easily accessible.
\end{abstract}

Keywords: Angiosperm, Dichotomous key, Egypt, Fossil wood, Wood anatomy.

\section{Introduction}

Identification of fossil wood to the species level has always been a difficult task as compared to modern wood. This is attributable foremost to the preservation state of fossil wood specimens as some specimens may not be in a sufficiently good condition to show important features. Some specimens may be so badly preserved that they cannot be identified to generic level or even assigned to a certain family. Another major difficulty is that some of the original descriptions of the taxa reported previously in the fossil flora of Egypt (e.g., Unger, 1823; Felix, 1883; Schenk, 1883; Stenzel, 1904; Kräusel \& Stromer, 1924; Kräusel, 1939; Süss, 1987; etc.) are not easily available. Therefore, this paper is in essence an attempt to provide an easily accessible alternative for the accurate determination of unknown fossil wood specimens that might be encountered in Egypt. It presents two identification keys to the validly published 19 Palmoxylon species and the 46 fossil dicot wood species that have so far been recorded from the Egyptian strata. Unpublished taxa or those identified only to the family level are excluded from the present keys. The unidentified species of Combretoxylon which was referred to by Boureau et al. (1983) from an unknown early Miocene locality in the Western desert without any description or illustrations is also omitted.

\section{Material and Methods}

Data in keys I and II are based on descriptions of fossil wood species in original literature: (Unger, 1823; Felix, 1883; Schenk, 1883; Stenzel, 1904; Kräusel \& Stromer, 1924; Bancroft, 1935; Kräusel, 1939; Sahni, 1964; Louvet, 1965; Müller-Stoll \& Mädel, 1967; Lemoigne \& Beauchamp, 1972; Mädel-Angeliewa \& Müller-Stoll, 1973; Prakash \& Bande, 1980; Süss, 1987; Cevallos-Ferriz \& Barajas-Morales, 1994; Mehrotra et al., 1999; ElSaadawi et al., 2011; El-Saadawi et al., 2017; NourEl-Deen et al., 2018) together with the additional references mentioned in Tables 1 and 2. Additional data was extracted from the insidewood database (InsideWood, 2004 onwards; Wheeler, 2011) for dicot wood species.

\section{Results}

The following are two strictly dichotomous bracketed keys for the 19 and 46 monocot and dicot wood species of Egypt, respectively:

"Corresponding author email: zainabelnoamani@yahoo.com

Received 6/1/ 2020; Accepted 3/2/ 2020

DOI: $10.21608 /$ ejbo.2020.22036.1431

Edited by: Prof. Dr. Adel El-Gazzar, Faculty of Science, El-Arish University, El-Arish, N. Sinai, Egypt.

(C)2020 National Information and Documentation Center (NIDOC) 


\section{Key I}

A dichotomous key to the 19 Palmoxylon species (monocots)

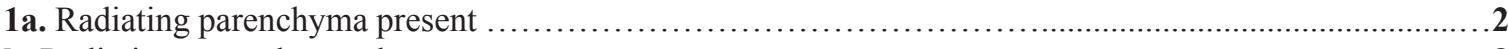

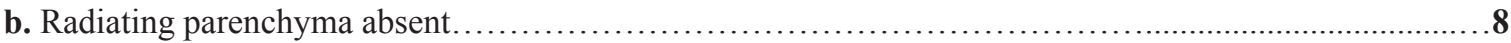

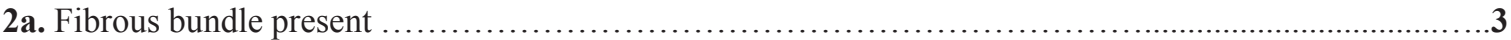

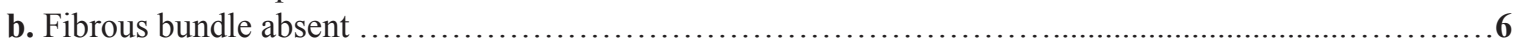

3a. Radiating parenchyma present around the whole bundle in an appendage-like manner ........................

Palmoxylon araneus Nour-El-Deen, El-Saadawi \& Thomas

b. Radiating parenchyma locally present either around the fibrous or vascular part...............................4

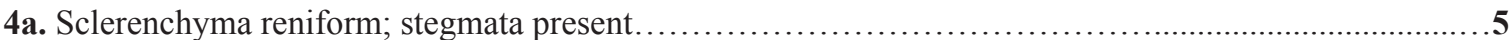

b. Sclerenchyma complanate; stegmata absent......................Palmoxylon lacunosum (Unger) Felix

5a. General ground tissue extremely lacunar, cells very elongate rod-like, thick-walled; tabular parenchyma developed around the whole bundle; sclerotic nests present

Palmoxylon qatraniense Nour-El-Deen, El-Saadawi \& Thomas

b. General ground tissue lacunar, cells trabecular, thin-walled; tabular parenchyma developed around the fibrous part; sclerotic nests absent

Palmoxylon elsaadawii Nour-El-Deen \& Thomas

6a. Tabular parenchyma present; vascular part exerted.....................................................

b. Tabular parenchyma absent; vascular part included or partly exerted........Palmoxylon deccanense Sahni

7a. General ground tissue slightly lacunar; tabular parenchyma 1-2 layers around the fibrous part and rarely over the whole bundle.............................................................................. Palmoxylon indicum Sahni

b. General ground tissue compact, cells of simple shape with small air spaces; tabular parenchyma 2-3 layers around the fibrous part

Palmoxylon sagari Sahni

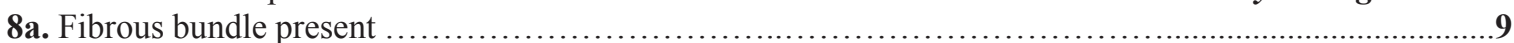

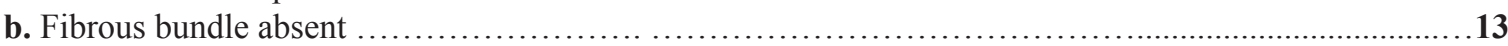

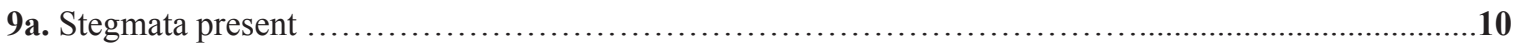

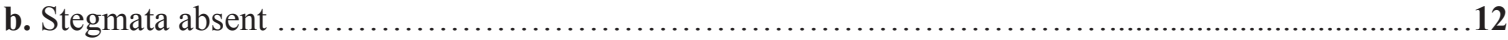

10a. Sclerenchyma cordate; tabular parenchyma 2-3 layers around the whole bundle

Palmoxylon prismaticum Sahni

b. Sclerenchyma lunate or lunate reniform; tabular parenchyma slightly developed either around the fibrous or vascular part

11a. Fibrous bundles numerous; tabular parenchyma well-developed around the vascular part

Palmoxylon zittelii Schenk

b. Fibrous bundles few; tabular parenchyma slightly developed around the fibrous part.....

Palmoxylon pondicherriense Sahni

12a. Sclerenchyma complanate; general ground tissue compact ..................Palmoxylon edwardsi Sahni b. Sclerenchyma vaginate; general ground tissue lacunar ............................ Palmoxylon stromeri Kräusel

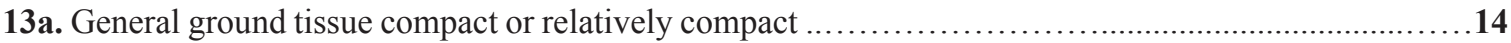

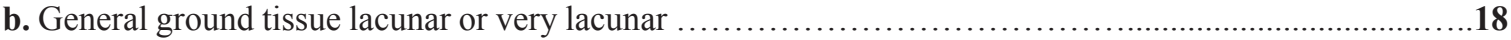

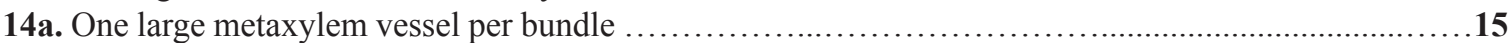

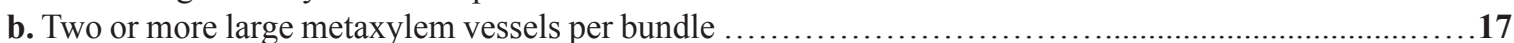

15a. Sclerenchyma sagittate; diminutive fibrovascular bundles commonly scattered especially in the central zone; xylem exerted as a long narrow tongue-like process. ...

Palmoxylon pyriforme Sahni

b. Sclerenchyma lunate or cordate; diminutive fibrovascular bundles absent or less frequent...................16

16a. Sclerenchyma lunate; general ground tissue relatively compact with small air spaces; tabular parenchyma multilayered around the vascular part

Palmoxylon aschersonii Schenk

b. Sclerenchyma cordate; general ground tissue compact; tabular parenchyma 2-3 layers around the fibrous part

Palmoxylon compactum Sahni

17a. Sclerenchyma reniform, cordate and occasionally sagittate; xylem vessels included or partially exerted; tabular parenchyma 1-2 layers around the fibrous part........................... Palmoxylon rewahense Sahni b. Sclerenchyma reniform; xylem vessels at least two (up to 4), exerted; tabular parenchyma multilayered around the vascular part

Palmoxylon libycum (Stenzel) Kräusel

18a. Sclerenchyma cordate; general ground tissue very lacunar, cells thin-walled with regular geometrical (Y or V) shapes; tabular parenchyma 1-2 layers around the fibrous part, interrupted by air spaces

Palmoxylon geometricum Sahni 
b. Sclerenchyma reniform; general ground tissue lacunar, cells of simple shapes and straight walls, scattered thick-walled cells present; tabular parenchyma several layers around the fibrous part.

Palmoxylon wadiai Sahni

\section{Key II}

A dichotomous key to the 46 dicotyledonous fossil wood species

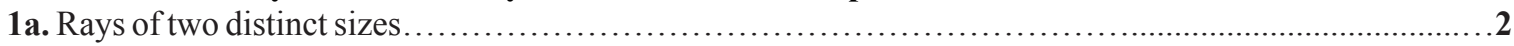

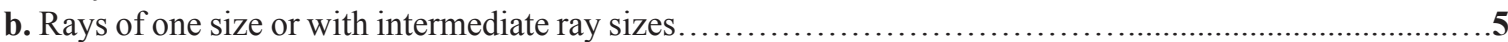

2a. Wood ring-porous.......................................................ercoxylon retzianum Kräusel

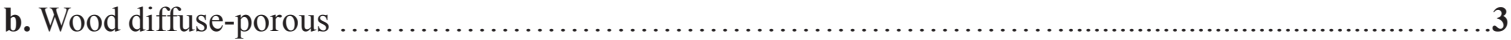

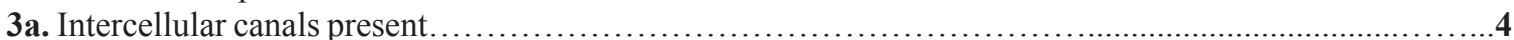

b. Intercellular canals absent........................................... Tamaricoxylon africanum (Kräusel) Boureau

4a. Vessel clusters common.......................................................... Proteoxylon chargeense Kräusel

b. Vessels solitary or in radial multiples of 2-3...................................... Hibiscoxylon niloticum Kräusel

5a. Ray exclusively uniseriate, rarely with biseriate portions as wide as uniseriate portion or completely biseriate...6

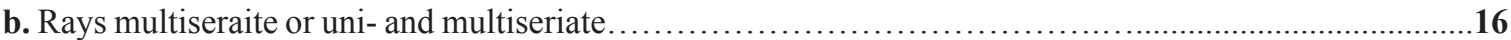

6a. Axial parenchyma absent or rare (scanty paratracheal as narrow incomplete sheath around the vessels or

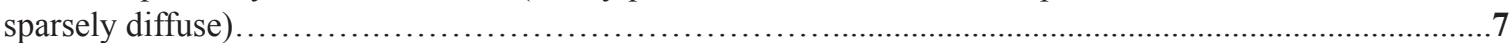

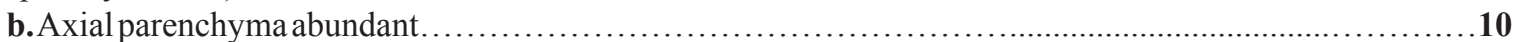

7a. Rays heterocellular with enlarged ray cells

Terminalioxylon intermedium (Kräusel) Mädel-Angeliewa \& Müller-Stoll

b. Rays homocellular, at most with upright marginal cells.................................................8

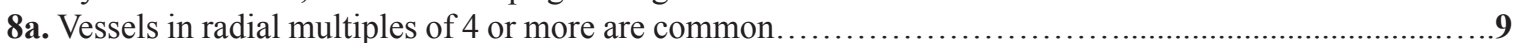

b. Vessels solitary and sometimes in short radial multiples of 2-3.........? Leguminoxylon albizziae Kräusel

9a. Parenchyma chambered, mostly with crystal..................................... Sapindoxylon stromeri Kräusel

b. Parenchyma non-chambered, without crystal. .............. Ebenoxylon ebenoides (Schenk) Edwards

10a. Diffuse and diffuse in aggregate apotracheal parenchyma abundant .......................................11

b. Diffuse and diffuse in aggregate apotracheal parenchyma absent or sparsely present.........................12

11a. Vessels predominantly solitary, $28-80 / \mathrm{mm}^{2}$.

? Celastrinoxylon sp. of Kräusel

b. Vessels solitary or in radial multiples of $2-8,20-32 / \mathrm{mm}^{2}$.

Ebenoxylon aegyptiacum Kräusel

12a. Axial parenchyma bands present.

b. Axial parenchyma bands absent or present only as discontinuous bands surrounding small secretory canals...4

13a. Parenchyma band wide ( $>3$ cells), forming curved bands.

Ficoxylon blanckenhorni Kräusel

b. Parenchyma band narrow (only 1-2 cells), at least in some zones

? Ficoxylon sp. of Kräusel

14a. Vessels $28-68 / \mathrm{mm}^{2}$, radial multiples of 4 or more are common

Terminalioxylon geinitzii (Schenk) Mädel-Angeliewa \& Müller-Stoll

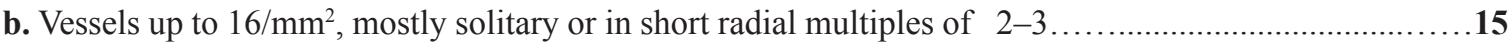

15a. Diffuse apotracheal parenchyma sparsely present ....

b. Diffuse apotracheal parenchyma absent

Terminalioxylon edwardsii (Kräusel) Mädel-Angeliewa \& Müller-Stoll

Terminalioxylon primigenium (Schenk) Mädel-Angeliewa \& Müller-Stoll

16a. Multiseriate rays at most 3 cells wide, exceptionally reach to 4 ............................................17

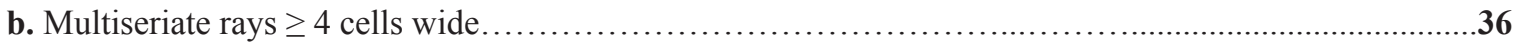

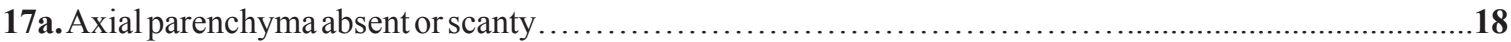

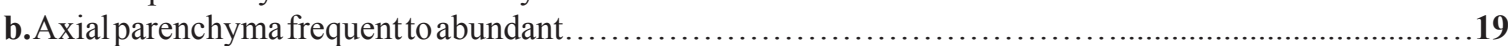

18a. Growth rings recognizable by the naked eye; rays homcellular, 6-40 cells high.

Atherospermoxylon aegyptiacum (Schenk) Kräusel

b. Growth rings indistinct; rays heterocellular, 6-12 (-25) cells high.

Schimoxylon dachalense (Kräusel) Kramer

19a. Apotracheal parenchyma abundant either diffuse or diffuse in aggregates...................................20

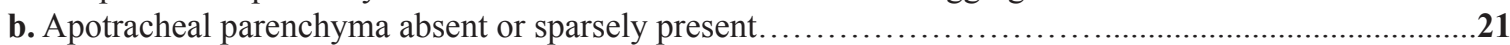

20a. Apotracheal parenchyma diffuse, paratracheal parenchyma vasicentric to aliform, confluent forming short irregular bands

Tetrapleuroxylon acaciae (Kräusel) Müller-Stoll \& Mädel

b. Apotracheal parenchyma diffuse in aggregates, paratracheal parenchyma absent or scanty 
Bombacoxylon owenii (Carruthers) Gottwald

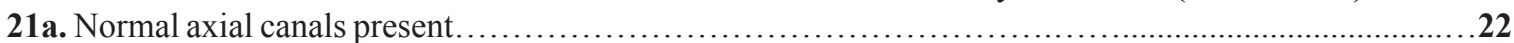

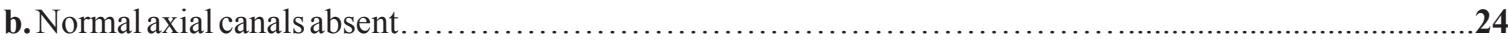

22a. Axial canals diffuse or in short tangential lines..................Dipterocarpoxylon africanum Bancroft

b. Axial canals concentric (in long tangential lines).....

23a. Paratracheal parenchyma lozenge-aliform, confluent; rays heterocellular

Copaiferoxylon matanzensis Cevallos-Ferriz \& Barajas-Morales

b. Paratracheal parenchyma vasicentric, slightly aliform, sometimes confluent; rays homocellular.

Copaiferoxylon migiurtinum (Chiarugi) Müller-Stoll \& Mädel

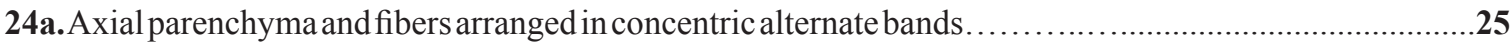

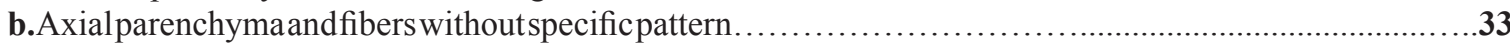

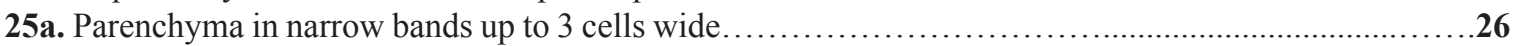

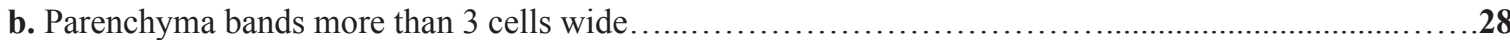

26a. Vessels $70-120 / \mathrm{mm}^{2}$

Celastrinoxylon celastroides (Schenk) Kräusel

b. Vessels up to $14 / \mathrm{mm}^{2}$

Euebenoxylon saharicum Süss

27a. Prismatic crystals present in chambered axial parenchyma cells Guttiferoxylon fareghense Kräusel

28a. Parenchyma bands wider than the alternating fiber bands, 6-25 cells wide.

Glutoxylon symphonioides (Bancroft) Lemoigne

b. Parenchyma bands narrower than or at most as wide as the alternating fiber bands, 3-9 cells wide..........29

29a. Fibers septate; tyloses present.

Ficoxylon cretaceum Schenk

b. Fibers non-septate; tyloses absent

.30

30a. Ray height $>1 \mathrm{~mm}$, up to 90 cells high... Cynometroxylon sp. cf. C. holdenii (Gupta) Prakash \& Bande

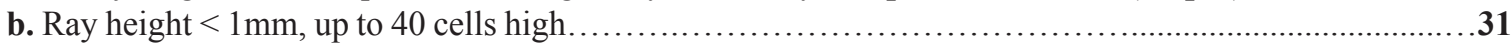

31a. Rays storied..

Cynometroxylon tunesense Delteil-Desneux

b. Rays non-storied.

32a. Vestured pits present.

Cynometroxylon holdenii (Gupta) Prakash \& Bande

b. Vestured pits absent

Cynometroxylon sp. of El-Saadawi et al.

33a. Axial parenchyma paratracheal as vasicentric, aliform, confluent; and also in irregular tangential bands...34 b. Axial parenchyma only paratracheal as vasicentric, aliform, sometimes confluent; tangential parenchyma absent or present only as marginal bands delineating the growth rings

34a. Ray height $>1 \mathrm{~mm}$, up to 100 (usually 30-40) cells high

Mimosoxylon calpocalycoides Lemoigne

Sterculioxylon giarabubense (Chiarugi) Kräusel

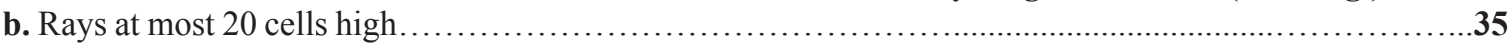

35a. Axial banded parenchyma mostly in narrow bands or lines up to 3 cells wide; prismatic crystals usually present either in ray or in axial parenchyma cells.........................? Leguminoxylon sp. of Kräusel b. Axial banded parenchyma mostly more than 3 cells wide; prismatic crystals absent

Dalbergioxylon dicorynioides Müller-Stoll \& Mädel

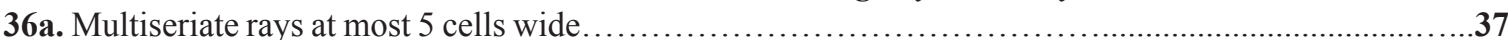

b. Multiseriate rays $>5$ cells wide

Cordioxylon barthelii Süss

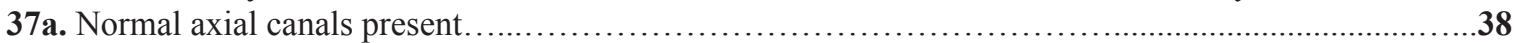

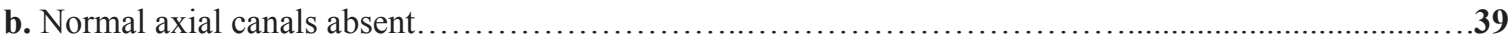

38a. Axial canals in concentric rings; axial parenchyma mostly paratracheal as vasicentric, aliform, in addition to marginal parenchyma bands surrounding axial canals............. Detarioxylon aegyptiacum (Unger) Louvet b. Axial canals diffuse; axial parenchyma forming irregular bands ( $2-8$ cells wide).

Andiroxylon aegyptiacum Ziada

39a. Axial Parenchyma not well-developed, only narrow vasicentric, weakly aliform or rarely confluent, sometimes narrow marginal parenchyma bands also present........................................................40 b. Axial Parenchyma more developed, broadly aliform, confluent or banded, sometimes apotracheal diffuse parenchyma also present.

40a. Axial Parenchyma only narrow vasicentric, rays 2-27 (mostly 15) cells high.

Mimosoxylon tenax (Felix) Müller-Stoll \& Mädel

b. Axial Parenchyma narrow vasicentric to weakly aliform, in addition to marginal parenchyma bands........41 
41a. Percentage of solitary vessels up to $70 \%$.

Dichrostachyoxylon palaeonyassanum Lakhanpal \& Prakash

b. Percentage of solitary vessels $50-55 \%$ ...42

42a. Tangential diameter of vessels $100-200 \mu$; prismatic crystals abundant in chambered axial parenchyma cells.

Dichrostachyoxylon zirkelii (Felix) Müller-Stoll \& Mädel

b. Tangential diameter of vessels $50-100 \mu$; prismatic crystals absent or less frequent

Dichrostachyoxylon royadeurm Privé

43a. Diffuse apotracheal parenchyma abundant

Tetrapleuroxylon zaccarinii (Chiarugi) Müller-Stoll \& Mädel

b. Diffuse apotracheal parenchyma absent.

44a. Axial banded parenchyma mostly in narrow bands or lines up to 3 cells wide; sometimes more than 3 cells wide; rays heterocellular. Acacioxylon vegae Schenk

b. Axial banded parenchyma present only as marginal bands delineating the growth rings; rays homocellular....45

45a. Paratracheal parenchyma lozenge-aliform and confluent. Afzelioxylon kiliani Louvet

b. Paratracheal parenchyma vasicentric, lozenge-aliform and confluent ....

Afzelioxylon welkitii (Lemoigne \& Beauchamp) Lemoigne

TABLE 1 . An alphabetical list of the 19 Palmoxylon species (Arecaceae) recorded so far from the Egyptian strata with their geological ages, data source reference(s) and numbers in key I.

\begin{tabular}{|c|c|c|c|}
\hline Palmoxylon species & Geological age & Reference & Key № \\
\hline $\begin{array}{l}\text { P. araneus Nour-El-Deen, El-Saadawi } \\
\& \text { Thomas }\end{array}$ & Oligo & Nour-El-Deen et al. (2018) & 3(a) \\
\hline P. aschersonii Schenk & $\begin{array}{l}\text { Oligo, Oligo/Mio, } \\
\text { Mio, Plio }\end{array}$ & $\begin{array}{l}\text { Stenzel (1904), Kräusel \& Stromer (1924), } \\
\text { El-Saadawi et al. (2002, 2010) }\end{array}$ & $16(a)$ \\
\hline P. compactum Sahni & Mio & Sahni (1964), El-Saadawi et al. (2004) & 16(b) \\
\hline P. deccanense Sahni & Mio & Sahni (1964), Kamal El-Din et al. (2013) & $6(b)$ \\
\hline P. edwardsi Sahni & Mio & Sahni (1964), Kamal El-Din et al. (2013) & $12(a)$ \\
\hline P. elsaadawii Nour-El-Deen \& Thomas & Oligo & Nour-El-Deen et al. (2018) & $5(b)$ \\
\hline P. geometricum Sahni & Oligo, Mio & Sahni (1964), El-Saadawi et al. (2004) & $18(a)$ \\
\hline P. indicum Sahni & Mio & Sahni (1964), El-Saadawi et al. (2004) & $7(\mathbf{a})$ \\
\hline P. lacunosum (Unger) Felix & Oligo, Mio & $\begin{array}{l}\text { Unger (1823), Felix (1883), Stenzel } \\
\text { (1904), Kräusel \& Stromer (1924) }\end{array}$ & $4(b)$ \\
\hline P. libycum (Stenzel) Kräusel & $\begin{array}{l}\text { Oligo, Oligo/Mio, } \\
\text { Mio }\end{array}$ & $\begin{array}{l}\text { Stenzel (1904), Kräusel \& Stromer (1924), } \\
\text { El-Saadawi et al. (2002) }\end{array}$ & 17(b) \\
\hline P. pondicherriense Sahni & Oligo, Mio & Sahni (1964), El-Saadawi et al. (2004) & $11(b)$ \\
\hline P. prismaticum Sahni & Mio & Sahni (1964), Kamal El-Din et al. (2013) & $10(a)$ \\
\hline P. pyriforme Sahni & Oligo, Mio & Sahni (1964), Kamal El-Din et al. (2013) & $15(a)$ \\
\hline $\begin{array}{l}\text { P. qatraniense Nour-El-Deen, El- } \\
\text { Saadawi \& Thomas }\end{array}$ & Oligo & Nour-El-Deen et al. (2018) & $5(a)$ \\
\hline P. rewahense Sahni & Mio & Sahni (1964), El-Saadawi et al. (2004) & 17(a) \\
\hline P. sagari Sahni & Mio & Sahni (1964), Kamal El-Din et al. (2013) & $7(b)$ \\
\hline P. stromeri Kräusel & Cretaceous & Kräusel \& Stromer (1924) & $12(b)$ \\
\hline P. wadiai Sahni & Mio & Sahni (1964), El-Saadawi et al. $(2004,2010)$ & $18(b)$ \\
\hline P. zittelii Schenk & Cretaceous & Stenzel (1904), Kräusel \& Stromer (1924) & 11(a) \\
\hline
\end{tabular}

Note: Mio $=$ Miocene, Oligo $=$ Oligocene, Plio $=$ Pliocene. 
TABLE 2. An alphabetical list of the 46 fossil dicot wood species recorded so far from the Egyptian strata with their possible botanical affinities, geological ages, data source reference(s) and numbers in key II.

Acacioxylon vegae Schenk
Afzelioxylon kiliani Louvet
Afzelioxylon welkitii (Lemoigne \&
Beauchamp) Lemoigne
Andiroxylon aegyptiacum Ziada
Atherospermoxylon aegyptiacum
(Schenk) Kräusel
Bombacoxylon owenii (Carruthers)
Gottwald
Celastrinoxylon celastroides
(Schenk) Kräusel
? Celastrinoxylon sp. of Kräusel
Copaiferoxylon matanzensis
Cevallos-Ferriz \& Barajas-Morales
Copaiferoxylon migiurtinum
(Chiarugi) Müller-Stoll \& Mädel
Cordioxylon barthelii Süss

Cynometroxylon holdenii (Gupta)

Prakash \& Bande

Cynometroxylon tunesense DelteilDesneux

Cynometroxylon sp. cf. C. holdenii (Gupta) Prakash \& Bande

Cynometroxylon sp. of El-Saadawi et al.

Dalbergioxylon dicorynioides Müller-Stoll \& Mädel

Detarioxylon aegyptiacum (Unger) Louvet

Dichrostachyoxylon palaeonyassanum Lakhanpal \& Prakash

Dichrostachyoxylon royaderum Privé

Dichrostachyoxylon zirkelii (Felix) Müller-Stoll \& Mädel

Dipterocarpoxylon africanum Bancroft

Ebenoxylon aegyptiacum Kräusel

Ebenoxylon ebenoides (Schenk)

Edwards

\begin{tabular}{|c|c|c|}
\hline Fabaceae & Oligo & Kräusel (1939) \\
\hline Fabaceae & Oligo & $\begin{array}{l}\text { Louvet (1965), El-Saadawi et al. } \\
\text { (2011) }\end{array}$ \\
\hline Fabaceae & Oligo, Mio & $\begin{array}{l}\text { Lemoigne \& Beauchamp (1972), } \\
\text { Kamal El-Din \& El-Saadawi } \\
\text { (2004) }\end{array}$ \\
\hline Fabaceae & Oligo & El-Saadawi et al. (2017) \\
\hline Monimiaceae & Oligo & Kräusel (1939) \\
\hline Malvaceae & $\begin{array}{l}\text { Oligo, } \\
\text { Oligo/Mio, } \\
\text { Mio, Plio, } \\
\text { Quaternary }\end{array}$ & $\begin{array}{l}\text { Kräusel (1939), El-Saadawi et al. } \\
\text { (2014b) }\end{array}$ \\
\hline Celastraceae & Cretaceous & $\begin{array}{l}\text { Kräusel (1939), Kamal El-Din } \\
\text { (2003) }\end{array}$ \\
\hline Celastraceae & Cretaceous & Kräusel (1939) \\
\hline Fabaceae & Oligo & $\begin{array}{l}\text { Cevallos-Ferriz \& Barajas-Morales } \\
\text { (1994), El-Saadawi et al. (2017) }\end{array}$ \\
\hline Fabaceae & Oligo & $\begin{array}{l}\text { Müller-Stoll \& Mädel (1967), El- } \\
\text { Saadawi et al. (2011) }\end{array}$ \\
\hline Boraginaceae & Palaeocene & Süss (1987) \\
\hline Fabaceae & Mio & $\begin{array}{l}\text { Prakash \& Bande (1980), Mehrotra } \\
\text { et al. (1999), Kamal El-Din \& El- } \\
\text { Saadawi (2004) }\end{array}$ \\
\hline Fabaceae & Oligo & El-Saadawi et al. (2011) \\
\hline Fabaceae & Mio & $\begin{array}{l}\text { Mehrotra et al. (1999), Kamal El- } \\
\text { Din et al. (2015) }\end{array}$ \\
\hline Fabaceae & Mio & El-Saadawi et al. (2014b) \\
\hline Fabaceae & $\begin{array}{l}\text { Oligo, } \\
\text { Oligo/Mio, } \\
\text { Mio }\end{array}$ & $\begin{array}{l}\text { Schenk (1883), Kräusel (1939), } \\
\text { Müller-Stoll \& Mädel (1967), El- } \\
\text { Saadawi et al. (2011) }\end{array}$ \\
\hline Fabaceae & $\begin{array}{l}\text { Eoc, Oligo, } \\
\text { Oligo/Mio, } \\
\text { Mio, Plio }\end{array}$ & $\begin{array}{l}\text { Kräusel (1939), Kamal El-Din \& } \\
\text { Refaat (2001) }\end{array}$ \\
\hline Fabaceae & Oligo & El-Saadawi et al. (2017) \\
\hline Fabaceae & Oligo & El-Saadawi et al. (2017) \\
\hline Fabaceae & Oligo & $\begin{array}{l}\text { Müller-Stoll \& Mädel (1967), El- } \\
\text { Saadawi et al. (2017) }\end{array}$ \\
\hline ipterocarpaceae & Mio & $\begin{array}{l}\text { Bancroft (1935), Kamal El-Din et } \\
\text { al. (2015) }\end{array}$ \\
\hline Ebenaceae & $\begin{array}{l}\text { Oligo, } \\
\text { Oligo/Mio }\end{array}$ & Kräusel (1939) \\
\hline Ebenaceae & Cretaceous & Kräusel (1939) \\
\hline Ebenaceae & Palaeogene & Süss (1987) \\
\hline
\end{tabular}

18(a) 
TABLE 2. Cont.

\begin{tabular}{|c|c|c|c|c|}
\hline Fossil taxa & Family & $\begin{array}{c}\text { Geological } \\
\text { age }\end{array}$ & Reference & Кey № \\
\hline Ficoxylon blanckenhorni Kräusel & Moraceae & $\begin{array}{l}\text { Oligo, } \\
\text { Oligo/Mio, } \\
\text { Mio }\end{array}$ & Kräusel (1939) & 13(a) \\
\hline Ficoxylon cretaceum Schenk & Moraceae & $\begin{array}{l}\text { Oligo, } \\
\text { Oligo/Mio, } \\
\text { Mio }\end{array}$ & $\begin{array}{l}\text { Schenk (1883), Kräusel (1939), } \\
\text { Kamal El-Din (2003) }\end{array}$ & 29(a) \\
\hline ? Ficoxylon sp. of Kräusel & Moraceae & Cretaceous & Kräusel (1939) & 13(b) \\
\hline $\begin{array}{l}\text { Glutoxylon symphonioides } \\
\text { (Bancroft) Lemoigne }\end{array}$ & Anacardiaceae & $\begin{array}{l}\text { Oligo/Mio, } \\
\text { Mio }\end{array}$ & Kräusel (1939) & 28(a) \\
\hline Guttiferoxylon fareghense Kräusel & Clusiaceae & Mio & Kräusel (1939) & 27(b) \\
\hline Hibiscoxylon niloticum Kräusel & Malvaceae & Cretaceous & Kräusel (1939) & $4(b)$ \\
\hline ? Leguminoxylon albizziae Kräusel & Fabaceae & Mio & Kräusel (1939) & $8(b)$ \\
\hline ? Leguminoxylon sp. of Kräusel & Fabaceae & Oligo & Kräusel (1939) & 35(a) \\
\hline $\begin{array}{l}\text { Mimosoxylon calpocalycoides } \\
\text { Lemoigne }\end{array}$ & Fabaceae & Oligo & El-Saadawi et al. (2011) & 33(b) \\
\hline $\begin{array}{l}\text { Mimosoxylon tenax (Felix) Müller- } \\
\text { Stoll \& Mädel }\end{array}$ & Fabaceae & Oligo, Mio & Müller-Stoll \& Mädel (1967) & $40(a)$ \\
\hline Proteoxylon chargeense Kräusel & Proteaceae & Cretaceous & Kräusel (1939) & 4(a) \\
\hline Quercoxylon retzianum Kräusel & Fagaceae & Oligo & Kräusel (1939) & 2(a) \\
\hline Sapindoxylon stromeri Kräusel & Sapindaceae & $\begin{array}{l}\text { Oligo, } \\
\text { Oligo/Mio, } \\
\text { Mio }\end{array}$ & $\begin{array}{l}\text { Kräusel (1939), El-Saadawi et al. } \\
\text { (2014a) }\end{array}$ & 9(a) \\
\hline $\begin{array}{l}\text { Schimoxylon dachalense (Kräusel) } \\
\text { Kramer }\end{array}$ & Theaceae & Cretaceous & Kräusel (1939) & $18(b)$ \\
\hline $\begin{array}{l}\text { Sterculioxylon giarabubense } \\
\text { (Chiarugi) Kräusel }\end{array}$ & Malvaceae & Oligo, Mio & Kräusel (1939) & 34(a) \\
\hline $\begin{array}{l}\text { Tamaricoxylon africanum } \\
\text { (Kräusel) Boureau }\end{array}$ & Tamaricaceae & Oligo & Kräusel (1939) & 3(b) \\
\hline $\begin{array}{l}\text { Terminalioxylon edwardsii } \\
\text { (Kräusel) Mädel-Angeliewa \& } \\
\text { Müller-Stoll }\end{array}$ & Combretaceae & $\begin{array}{l}\text { Oligo, } \\
\text { Oligo/Mio, } \\
\text { Mio }\end{array}$ & $\begin{array}{l}\text { Kräusel (1939), Mädel-Angeliewa } \\
\& \text { Müller-Stoll (1973), El-Saadawi } \\
\text { et al. (2013) }\end{array}$ & $15(a)$ \\
\hline $\begin{array}{l}\text { Terminalioxylon geinitzii (Schenk) } \\
\text { Mädel-Angeliewa \& Müller-Stoll }\end{array}$ & Combretaceae & Oligo, Mio & $\begin{array}{l}\text { Kräusel (1939), Mädel-Angeliewa } \\
\text { \& Müller-Stoll (1973), El-Saadawi } \\
\text { \& Kamal El-Din (2004) }\end{array}$ & $14(a)$ \\
\hline $\begin{array}{l}\text { Terminalioxylon intermedium } \\
\text { (Kräusel) Mädel-Angeliewa \& } \\
\text { Müller-Stoll }\end{array}$ & Combretaceae & $\begin{array}{l}\text { Cretaceous, } \\
\text { Oligo }\end{array}$ & $\begin{array}{l}\text { Kräusel (1939), Mädel-Angeliewa } \\
\text { \& Müller-Stoll (1973), El-Saadawi } \\
\text { \& Kamal El-Din (2004) }\end{array}$ & $7(a)$ \\
\hline $\begin{array}{l}\text { Terminalioxylon primigenium } \\
\text { (Schenk) Mädel-Angeliewa \& } \\
\text { Müller-Stoll }\end{array}$ & Combretaceae & $\begin{array}{l}\text { Oligo, } \\
\text { Oligo/Mio }\end{array}$ & $\begin{array}{l}\text { Kräusel (1939), Mädel-Angeliewa } \\
\text { \& Müller-Stoll (1973) }\end{array}$ & $15(b)$ \\
\hline $\begin{array}{l}\text { Tetrapleuroxylon acaciae } \\
\text { (Kräusel) Müller-Stoll \& Mädel }\end{array}$ & Fabaceae & $\begin{array}{l}\text { Oligo, } \\
\text { Oligo/Mio, } \\
\text { Mio }\end{array}$ & $\begin{array}{l}\text { Kräusel (1939), Müller-Stoll \& } \\
\text { Mädel (1967) }\end{array}$ & $20(a)$ \\
\hline $\begin{array}{l}\text { Tetrapleuroxylon zaccarinii } \\
\text { (Chiarugi) Müller-Stoll \& Mädel }\end{array}$ & Fabaceae & Oligo & $\begin{array}{l}\text { Müller-Stoll \& Mädel (1967), El- } \\
\text { Saadawi et al. (2011) }\end{array}$ & 43(a) \\
\hline
\end{tabular}

Note: Eoc $=$ Eocene, Mio $=$ Miocene, Oligo $=$ Oligocene, Plio $=$ Pliocene. 


\section{Discussion}

The two keys provided in this article help to facilitate the tentative allocation of an unknown fossil angiosperm wood specimen to an appropriate species using simple dichotomous keys, thus saving researchers precious time and providing them with easily accessible and accurate mean of identifying fossil angiosperm wood. With the two provided keys, students of fossil wood anatomy seeking to identify unknown specimens will no longer have to search for the protologues and/or diagnostic illustrations of individual taxa in rare and outdated literature.

The provided keys follow the standard dichotomous conventional format in which characters are sorted in a sequential order. They are mainly dependent, as much as possible, on the most distinctive, unambiguous and constant qualitative features of high taxonomic weight and avoid too much focus on quantitative features which are of variable nature. However, after the preliminary identification, it is advisable to proceed to the next confirming step by returning to more comprehensive catalogues providing full generic and specific descriptions.

A major advantage of Key I and II is that they comprise collectively all 65 fully recognized angiosperm fossil wood taxa reported so far from Egypt. Therefore, if any unknown specimen of fossil wood is uncovered locally in the future proves difficult to identify with the help of these two keys, it stands a good chance of belonging either to a new record to the fossil flora of Egypt or to a taxon new to science pending a more detailed study of it.

\section{References}

Bancroft, H. (1935) Some fossil dicotyledonous woods from Mount Elgon, East Africa. I. American Journal of Botany, 22, 164-183.

Boureau, E., Cheboldaeff-Salard, M., Koeniguer, J.C., Louvet, P. (1983) Evolution des flores et de la végétation tertiaires en Afrique, au nord de l' Equateur. Bothalia, 14 (3/4), 355-367.

Cevallos-Ferriz, S.R.S., Barajas-Morales, J. (1994) Fossil woods from the El Cien formation in Baja California sur: Leguminosae. IAWA Bulletin, 15, 229-245
El-Saadawi, W.E., Kamal El-Din, M. (2004) Terminalioxylon species from Gebel Qatrani Formation at Widan-el-Faras, Fayum, Egypt. Taeckholmia, 24(1), 63-78.

El-Saadawi, W.E., Youssef, S.G.M., Kamal El-Din, M.M. (2002) Fossil palm woods of Egypt. I. Palmoxylon aschersoni Schenk and P. libycum (Stenzel) Krausel. Taeckholmia, 22(2), 143-153.

El-Saadawi, W.E., Youssef, S.G.M., Kamal El-Din, M.M. (2004) Fossil palm woods of Egypt. II. Seven Tertiary Palmoxylon species new to the country. Review of Palaeobotany and Palynology, 129(4), 199-211.

El-Saadawi, W.E., Kamal El-Din, M.M., El-Faramawi, M., El-Noamani, Z.M. (2010) Fossil palm woods of Egypt. III. A new site for Palmoxylon aschersoni Schenk. and P. wadiai Sahni. Taeckholmia, 30, 145159.

El-Saadawi, W.E., Kamal El-Din, M.M., Attia, Y., ElFaramawi, M. (2011) The wood flora of the Cairo Petrified Forest, with five Paleogene new legume records for Egypt. Review of Palaeobotany and Palynology, 167, 184-195.

El-Saadawi, W.E., Kamal El-Din, M.M., El-Faramawi, M., Ziada, N.A. (2013) Terminalioxylon edwardsii from the Oligocene of Egypt, with a review of the genus in Africa. Taeckholmia, 33(4), 47-63.

El-Saadawi, W.E., Kamal El-Din, M., Darwish, M.H., Osman, R. (2014a) African Miocene dicot woods with two new records for this epoch from Egypt. Taeckholmia, 34, 1-24.

El-Saadawi, W.E., Kamal El-Din, M.M., Wheeler, E., Osman, R., El-Faramawi, M., El-Noamani, Z.M. (2014b) Early Miocene woods of Egypt. IAWA Journal, 35(1), 35-50.

El-Saadawi, W.E., Ziada, N.A., El-Faramawi, M., Kamal El-Din, M.M., Loutfy, M.H.A. (2017) The Cairo Petrified Forest revisited. Review of Palaeobotany and Palynology, 238, 34-42.

Felix, J. (1883) Die fossilen Hölzer Westindiens. Sammlung palaeontologischer Abhandlungen. Serie I, Heft 1. Verlag von T. Fischer, Cassel, 29pp.

Insidewood (2004-onwards) http://insidewood.lib.ncsu. edu/search. (accessed November-December 2019) 
Kamal El-Din, M.M. (2003) Petrified wood from the Farafra Oasis, Egypt. IAWA Journal, 24(2), 163-172.

Kamal El-Din, M.M., Refaat, A.A. (2001) Detarioxylon aegyptiacum (Leguminosae) from Lower Miocene marine sediments in Southern Sinai. Taeckholmia, 21(1), 103-114.

Kamal El-Din, M.M., El-Saadawi, W.E. (2004) Two Leguminosae woods from the Miocene of Gebel Ruzza, Egypt. IAWA Journal, 25(4), 471-483.

Kamal El-Din, M.M., Darwish, M.H., El-Saadawi, W.E. (2013) Fossil palm woods of Egypt: IV. New Palmoxylon records with a summary on macrofossil African Arecaceae. Palaeontographica Abt. B., 290(1-3), 41-61.

Kamal El-Din, M.M., Darwish, M.H., El-Saadawi, W.E. (2015) Novelties on Miocene woods from Egypt with a summary on African fossil woods of Fabaceae, Malvaceae and Dipterocarpaceae. Palaeontographica Abt. B., 292(4-6), 173-199.

Kräusel, R. (1939) Ergebnisse der Forschungsreisen Prof. E. Stromers in den Wüsten Ägyptens, IV. Die fossilen Floren Ägyptens 3. Die fossilen Pflanzen Ägyptens, E-L. Abhandlungen der Bayerischen Akademie der Wissenschaften Mathematisch Abt. (N.F.), 47, 1-140.

Kräusel, R., Stromer, E. (1924) Ergebnisse der Forschungsreisen Prof. E. Stromers in den Wüsten Ägyptens, IV. Die fossilen Floren Ägyptens 1-3. A-C. Abhandlungen der Bayerischen Akademie der Wissenschaften Mathematisch Abt., 30(2), 1-48.

Lemoigne, Y., Beauchamp, J. (1972) Paléoflores tertiaires de la region de Welkite (Ethiopie, Preovince du Shoa). Bulletin de Société Géologique de France, 14, 336-346.

Louvet, P. (1965) Sur une Légumineuse fossile nouvelle de Tinrhert (Algérie): Afzelioxylon kiliani n.g., n.sp. Actes du 90e Congrès national des sociétés savantes, Nice, Section des Sciences, Comptes rendus, 2, $317-$ 332.

Mädel-Angeliewa, E., Müller-Stoll, W.R. (1973) Kritische Studien über fossile CombretaceenHölzer: über Hölzer vom Typus Terminalioxylon
G. Schönfeld mit einer Revision der bisher $\mathrm{zu}$ Evodioxylon Chiarugi gestellten Arten. Palaeontographica Abt. B., 142(4/6), 117-136.

Mehrotra, R.C., Awasthi, N., Dutta, S.K. (1999) Study of fossil wood from the upper Tertiary sediments (Siwalik) of Arunachal Pradesh, India and its implications in palaeoecological and phytogeographical interpretations. Review of Palaeobotany and Palynology, 107(3-4), 223-247.

Müller-Stoll, W.R., Mädel, E. (1967) Die fossilen Leguminosen-Hölzer. Eine Revision der mit Leguminosen verglichenen fossilen Hölzer und Beschreibungen älterer und neuer Arten. Palaeontographica Abt. B., 119, 95-174.

Nour-El-Deen, S., Thomas, R., El-Saadawi, W.E. (2018) First record of fossil Trachycarpeae in Africa: Three new species of Palmoxylon from the Oligocene (Rupelian) Gebel Qatrani Formation, Fayum, Egypt. Journal of Systematic Palaeontology, 16(9), 741766.

Prakash, U., Bande, M.B. (1980) Some more fossil woods from the Tertiary of Burma. Palaeobotanist, 26, 261-278.

Sahni, B. (1964) Revisions of Indian fossil plants: part III. Monocotyledons. Monogr. Birbal Sahni Inst. Palaeobotanist, 1, 1-89.

Schenk, A. (1883) Fossile Hölzer. Palaeontographica Abt. B., 30(2), 1-19.

Stenzel, K.G. (1904) Fossile Palmenhölzer. Beiträge zur Paläontologie und Geologie Österreich-Ungarns und des Orients, 16, 107-287. Wien.

Süss, H. (1987) Zwei neue kieselhölzer, Cordioxylon barthelii gen. nov., sp. nov., und Euebenoxylon saharicum gen. nov., sp. nov., aus der Südwestsahara, Ägypten. Feddes Repertorium, 98(9-10), 521-536.

Unger, F. (1823) De palmie fossilibus. pp. 53-70 in C.F.P. Martius Historia naturalis palmarum. Weigel, Lipsiae.

Wheeler, E.A. (2011) InsideWood - A web resource for hardwood anatomy. IAWA Journal, 32, 199-211. 


\section{الخشب الأحفوري المصري من كاسيات البذور: دليل التعريف اعتماداءعلي الصفات التشريحية}

\section{زينب محمد النعماني}

معمل السعداوي ـ قسم النبات ـ كلية العلوم - جامعة عين شمس ـ القاهرة ـ مصر.

استهدف هذا البحث تصميم مفتاحين تعريفيين لأنواع الخشب المتحجر التابعة لكاسيات البذور والتي تم تسجيلها حتي الآن من مصر وذللك من أجل تسهيل التعريف المبئي لأي عينة غيرمعرفة من الخشب المتحجر بالطبقات الرسوبية المصرية وتنتمي إلى كاسيات البذور. أنتنمل الففتاح الأول على عدد 19 نوعاء من جنس Palmoxylon وهو جنس شكلي لجذوع النخيل المتحجر و الذي ينتمي إلى النباتات ذوات الفلقة الواحدة ويتمثل بوفرة في الطبقات الرسوبية

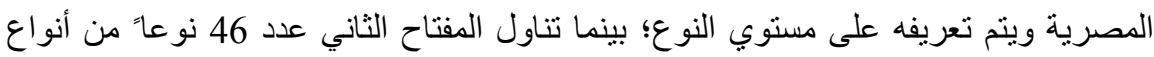
الخشب المتحجر التابعة للنباتات ذوات الفلقتين و التي تتنمي لفصائل متعددة وسجلت من أعمار جيولوجية ومو اقع مصرية مختلفة. تعود مثل هذه المفاتيح التعريفية بالنفع على دارسي الحفريات

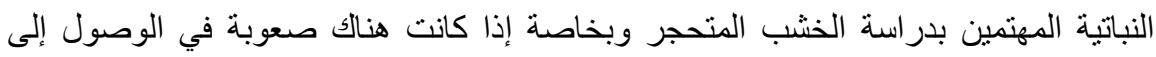
المر اجع الأصلية الخاصة بتعريف مثل هذه الأنواع المسجلة قديماً. 